\section{DOMAIN STRUCTURES IN CO/Pd MULTILAYERS}

V. Kamberský, P. de Haan, J.C. Lodder, J. Šimšová*, R.Gemperle*

MESA, University of Twente, Box 217, 7500 AE, Enschede, NL

*Institute of Physics, CAS, Cukrovarnická 10,16200 Prahal0, CS

Potential application of mutilayers (ML) in magneto-optical recording is connected with the problem of information stability, which in turn mostly depends on the coercivity but also on the magnetostatic forces in metastable domain configurations. Our aim is to discuss the periodic domain model for ML [1-3] and to compare its predictions with domain observation, the slopes of M-H loops and the anisotropy constants of medium and high coercivity $\mathrm{Co} / \mathrm{Pd} \mathrm{ML}$

The periodic stripe model [1-3] balances the magnetostatic forces with surface tension in domain walls. As seen in Fig.1, we find that it predicts a non-monotonous dependence of the equilibrium stripe period $\mathrm{P}$ on the total ML thickness $T$, or the individual magnetic layer thickness $t$ if they decrease proportionally at constant number of layers $\mathrm{N}$ and constant propotion $\gamma$ of tot the magnetic volume: $\gamma=\mathrm{T} / \mathrm{Nt}$. To understand this behavior we plot in Fig. 2 the normalized magnetostatic energy density $e=2 E / \mu_{0} M_{s}^{2}[2,3]$ and the average magnetostatic force per unit wall area $\Phi=\mathrm{P} \partial \mathrm{e} / \partial \mathrm{P}$ as functions of the domain period realative to $\mathrm{t}$ and $\mathrm{T}$. At balance, $\Phi=2 \lambda \mathrm{P}$ if $\lambda=2 \sigma / \mu_{0} M_{s}^{2}, \sigma$ is the wall energy density. As indicated by the different scales in Figs.2a,b, the low-P and high-P maxima in $\Phi$ are shape resonance effects around $P \approx t$ and $P \approx T$, resp. In Fig 3 we plot the part of $\Phi$ due to inter-layer forces alone: around $P \approx T$ this is the main part of $\Phi$ which tends to shrink the domains, while at higher T/P it has the opposite tendency. Prevalence of
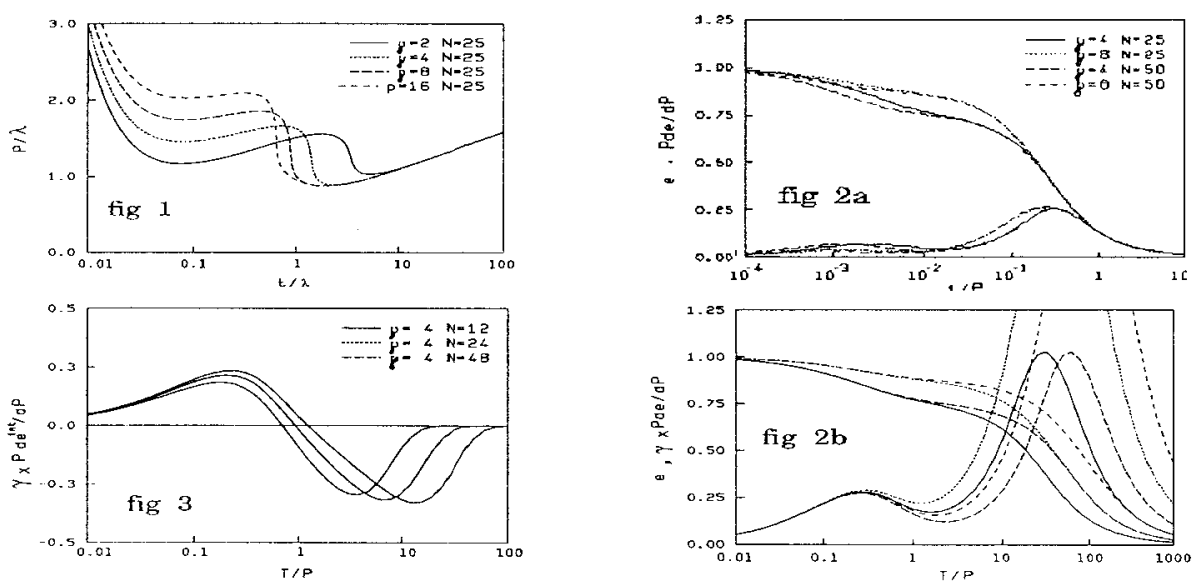

minimum equilib. $P$ ) occurs at $T \cong 2 \gamma \lambda$ (with $P \cong 7 \gamma \lambda$ ), which would also be predicted for a single layer of thickness $\mathrm{T}$, mean magnetization $\mathrm{M}_{S} / \gamma$ and accordingly diluted mean wall energy $\sigma / \gamma$.
We have also computed the initial slopes of $\mathrm{M}(\mathrm{H})$ curves from the model 12,31. Comparison of the computed and observed domain periods as well as of the $M(H)$ slopes around $M=0$ allows to estimate the domain wall energy.

Samples of $\mathrm{Co} / \mathrm{Pd} \mathrm{ML}$ were prepared by if sputtering in $\mathrm{Ar} \mathrm{M}-\mathrm{H}$ loops were measured by VSM and anisotropy constants $K_{\text {ef }}$ by a torque magnetometer [4]. Domain structures were studied using the Bitter-colloid patterns observed by SEM, on the M-H loops and on minor loops after a number of cycles. Examples of the observed domain structures are in Figs. 4 a,b for a medium and high coercitivity sample, resp. The sample parameters and preliminary results are in Table 1 :

\begin{tabular}{|c|c|c|c|c|c|c|c|c|c|c|c|c|}
\hline & $\mathrm{t}_{\mathrm{C} n}$ & $\mathrm{~T}$ & $\gamma$ & $\mathrm{M}_{\mathrm{S}}$ & $\mathrm{K}_{\mathrm{ef}}$ & $\mathrm{K}_{\mathrm{u}}$ & $\mathrm{dH} / \mathrm{dM}$ & $\mathrm{H}_{\mathrm{C}}$ & $\mathrm{P}$ & $\lambda$ & $\sigma_{\mathrm{P}}$ & $\sigma_{\mathrm{K}}$ \\
\hline & $\mathrm{nm}$ & $\mathrm{nm}$ & & $\mathrm{MA} / \mathrm{m}$ & \multicolumn{2}{|c|}{$\mathrm{MJm}^{-3}$} & & $\mathrm{kA} / \mathrm{m}$ & $\mathrm{nm}$ & $\mathrm{nm}$ & \multicolumn{2}{|c|}{$\mathrm{mJm}^{-2}$} \\
\hline $\mathbf{a}$ & 0.85 & 57.4 & 2.7 & 1.85 & 0.4 & 2.5 & 0.21 & 36 & $70-100$ & $3-4$ & 6 & 20 \\
\hline $\mathrm{b}$ & 0.43 & 83.9 & 7.9 & 1.6 & 1.6 & 3.2 & 0.05 & 110 & $250-300$ & $4.5-5.5$ & 8 & 22 \\
\hline
\end{tabular}

Here $\mathrm{K}_{\mathrm{u}}=\mathrm{K}_{\mathrm{ef}}-\mu_{0} \mathrm{M}_{\mathrm{s}}^{2} / 2$, both $\mathrm{K}_{\mathrm{u}}$ and $\mathrm{M}_{\mathrm{s}}$ refer to Co volume (25 layers). The mean domain period 政

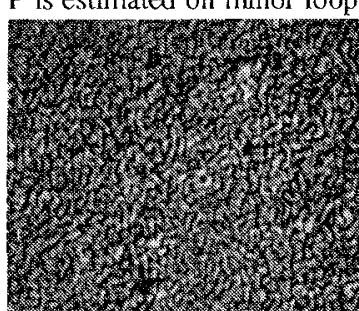

fig. 4 a $\mathrm{pl} \longrightarrow 1 \mathrm{~km}$

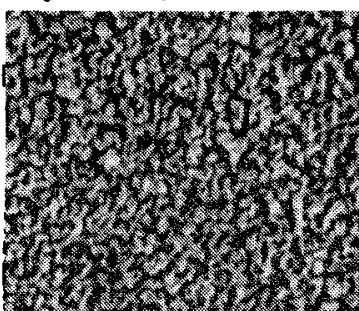
using the experimental T/P ratio and the model Domains in the "hard" sample (b) are apparently very close to the minimum period predicted by the model. The estimate of $\lambda$ agrees within the large uncertainty limits with that obtained comparing the measured and computed $\mathrm{dH} / \mathrm{dM}$ at $\mathrm{H}=\mathrm{H}_{c}$. Finally, $\sigma_{\mathrm{P}}$ is the wall energy density estimated as $\lambda\left(\mu_{0} M_{s}{ }^{2} / 2\right)$ from the domain period, while $\sigma_{K}=4\left(\mathrm{AK}_{\mathrm{u}}\right)^{1 / 2}$ is the classical energy density expected using the measured $K_{u}$ and estimated $A=10^{-11} \mathrm{~J} / \mathrm{m}^{3}$. The experimental values are considerably higher than those reported previously [2.3] but still much lower than the expectation based on $\mathrm{K}_{\mathrm{U}}$ abon $[2,3]$ but still mu lower the onfiguration should partly correct this discrepancy.

\section{References:}

A.Suna: J.Appl.Phys. 59, 313 (1986)

H.J.G.Draaisma, W.J.M.de Jonge: J.Appl.Phys. 62, 3318 (1987)

W.R.Bennett C.D England D C.Person, C.M.Falco: J Appl.Phys, 69,4384 (9) P.d)

P.de Haan, Q.Meng, T.Katayama, J.C.Lodder, Journ.Mag.Mag.Mater.113 (1992) 